\title{
Electronic analog of chiral metamaterial: Helicity-resolved filtering and focusing of Dirac fermions in thin films of topological materials
}

\author{
Lu Zhao, ${ }^{1,2, *}$ Jianfeng Wang, ${ }^{2}$ Junwei Liu, ${ }^{2,3}$ Yong Xu, ${ }^{4}$ Bing-Lin Gu, ${ }^{5}$ Qi-Kun Xue,,${ }^{2,6}$ and Wenhui Duan ${ }^{2,5,6, \dagger}$ \\ ${ }^{1}$ School of Physics, Beihang University, Beijing 100191, China \\ ${ }^{2}$ State Key Laboratory of Low-Dimensional Quantum Physics, Department of Physics, Tsinghua University, Beijing 100084, China \\ ${ }^{3}$ Department of Physics, Massachusetts Institute of Technology, Cambridge, Massachusetts 02139, USA \\ ${ }^{4}$ Department of Physics, McCullough Building, Stanford University, Stanford, California 94305-4045, USA \\ ${ }^{5}$ Institute for Advanced Study, Tsinghua University, Beijing 100084, China \\ ${ }^{6}$ Collaborative Innovation Center of Quantum Matter, Tsinghua University, Beijing 100084, China
}

(Received 26 March 2015; published 21 July 2015)

\begin{abstract}
Control over the helicity degree of freedom of Dirac fermions is identified in thin films of topological materials which act as a tunable "chiral-metamaterial-like" platform to tame left- and right-handed Dirac fermions in two dimensions. Using topological crystalline insulator $\mathrm{SnTe}(111)$ thin films as an example, we perform the firstprinciples calculations and show that giant helicity splitting in the band structures can be induced under moderate electric field. Based on this result, helicity-resolved functionalities, including pronounced electron dichroism, helicity switching, helical negative refraction, and birefraction, are demonstrated, where the intrahelical scattering always dominates over the interhelical one. Such intriguing control strategy for helical Dirac fermions may hold great promise for the applications of helicity-based electron optics and nanoelectronics.
\end{abstract}

DOI: 10.1103/PhysRevB.92.041408

PACS number(s): 72.25.-b, 71.15.Mb, 73.20.-r, 73.63.-b

The helical nature, reflecting the spin-momentum correlations under rotation, plays a vital role in determining the physical properties of particles. In optics, a fascinating route has been paved to control photon helicity with chiral metamaterials lacking mirror symmetry [1-4], which can selectively make negative refraction or pronounced dichroism for only one circularly polarized eigenwave (Fig. 1). However, although the helical nature of spin-half Dirac fermions is well known in quantum field theory [5], the question remains whether metamaterial-like systems can be achieved for Dirac fermions based on their helicity degree of freedom (Fig. 1). An affirmative answer to this question would not only increase our insight into the universality of fundamental helicity-related physics, but also establish a useful platform to manipulate Dirac fermions for helicity-based electronic applications.

The burgeoning world of topological materials has produced various symmetry-protected quantum matters with helical Dirac surface states spanning the bulk band gap, such as topological insulators (TIs) [6-8] and topological crystalline insulators (TCIs) [9,10]. Superior to relativistic Dirac systems in the realm of particle physics, such peculiar state offers great opportunities to explore helicity-related physics at low energy in a solid. This fact also suggests that topological materials might constitute a potential candidate for generating the electronic analog of photonic chiral metamaterial, which could resolve the left-handed $(\mathrm{LH})$ and right-handed $(\mathrm{RH})$ Dirac fermions in two dimensions.

To this end, we consider the coupled helical surface state system in a thin-film geometry of topological materials in this work. In thin film, two surface Dirac cones located on the top and bottom surfaces are mirror images of each other obeying inversion symmetry and have opposite helicity

*zhaol@buaa.edu.cn

${ }^{\dagger}$ dwh@phys.tsinghua.edu.cn
[Fig. 2(a)]. Due to intersurface coupling, the two Dirac cones interact with each other, opening a hybridization gap [11-13]. More significantly, this feature allows the control over the helicity degree of freedom of Dirac fermions. First, the twofold helicity-degenerate band structures simultaneously sustain both LH and RH electron (or hole) states of Dirac fermions in the same system. Further, the helicity degeneracy can be removed in a controllable way by breaking the spatial inversion symmetry of the film, e.g., by applying an external electric field [14-16] or by using surface functionalization $[17,18]$ [Fig. 2(a)]. These facts form the basis for manipulating helical Dirac fermions in a manner similar to the manipulation of $\mathrm{LH}$ and $\mathrm{RH}$ photons in chiral metamaterials.

From a practical standpoint, the topological materials more sensitive to external electric field are more suitable to implement tunable helicity-based applications. Recent experimental development has confirmed the existence of TCI phase protected by crystalline symmetry [19-23], where TCI thin films may have the potential to create topological devices particularly susceptible to external electric field [24]. More recently, the (111)-oriented surfaces and films of TCI SnTe and related alloys have been extensively studied [25-31], which could offer a promising platform in its own right towards experimental realization of gate-tuned helicity-resolved physics for Dirac fermions.

Based on the density-functional theory (DFT) method [32-37], we calculate the electronic structures of $\mathrm{SnTe}(111)$ thin films with different thicknesses [38,39], where hydrogen saturation is adopted to stabilize the surfaces [27]. Using the Te-terminated film with 25 atomic layers as an example [Fig. 2(b)], Fig. 2(c) illustrates the helicity-degenerate band structure in the vicinity of the $\bar{\Gamma}$ point without electric field, while in Fig. 2(d) giant helicity splitting $\left(\Delta_{D} \sim 0.15 \mathrm{eV}\right)$ can be induced by a moderate electric field $\left(E_{\mathrm{ex}}=8.5 \mathrm{mV} / \AA\right)$ in the thin film, which is highly desired for thin-film device applications. The long-wavelength dynamics of Dirac fermions near 
(a)

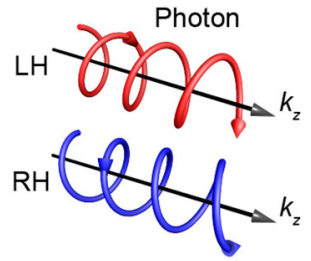

(b)

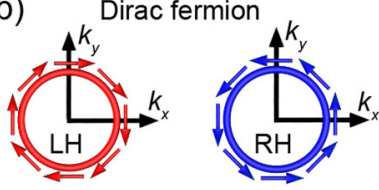

(c) Chiral negative refraction

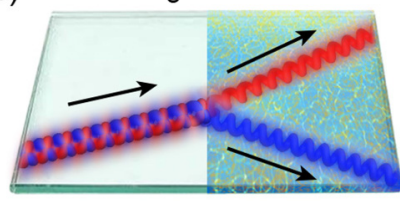

(d) Pronounced circular dichroism

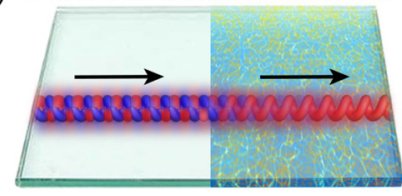

FIG. 1. (Color online) Analogy of helical properties between photons and Dirac fermions. (a) Left-handed (LH) and right-handed (RH) circular polarizations of photons. (b) LH and RH electron (or hole) states of Dirac fermions with opposite helical spin textures. Also shown are the chiroptical effects in chiral metamaterial. Only one circularly polarized eigenwave can be negatively refracted (c) or pronouncedly diminished (d). As an analogy, whether similar effects can happen for Dirac fermions, however, remains elusive.

the $\bar{\Gamma}$ point can be described by a four-band $\mathbf{k} \cdot \mathbf{p}$ Hamiltonian

$$
H_{0}=\hbar v_{F}[(\boldsymbol{\sigma} \times \boldsymbol{k}) \cdot \hat{z}] \tau_{z}+m \tau_{x}+\delta \tau_{z}+V_{0},
$$

where $\hbar$ is the reduced Planck's constant, $v_{F}$ is the Fermi velocity, $\sigma$ and $\tau$ are Pauli matrices representing spin and surfaces, respectively, $m$ describes the interaction between the top and bottom surface states due to the coupling effect, $\delta$ denotes the intersurface bias depending on the strength and polarity of the electric field, and $V_{0}$ stands for the local potential height. In Fig. 2(a), the band gap is given by $\Delta_{g}=2|m|$ and the energy difference between two Dirac points is defined by $\Delta_{D}=2 \sqrt{\delta^{2}+m^{2}}$ which characterizes the gate-tuned helicity splitting in the band structure. We can fit the material parameters in Eq. (1) as $m=29 \mathrm{meV}$, and $v_{F}=4.6 \times 10^{5} \mathrm{~m} / \mathrm{s}$, and $\delta= \pm 68 \mathrm{meV}$ for the fields along the $\pm z$ directions [Fig. 2(d)].

Most importantly, the removal of helicity degeneracy (i.e., $\delta \neq 0$ ) could manifest high tunability under perpendicular electric field, thus facilitating helicity-resolved control of Dirac fermions. For example, because the electric field directly shifts the helical Dirac cones in opposite directions in energy, moderate field strength may generate giant helicity splitting simultaneously for both electron and hole excitations interconnected by the Dirac-type equation (1), thus preserving the charge-conjugation symmetry. In contrast, such mechanism is essentially different from that of conventional Rashba effect, where the electric field plays a crucial role in producing the extrinsic spin-orbit coupling [40]. Additionally, if the
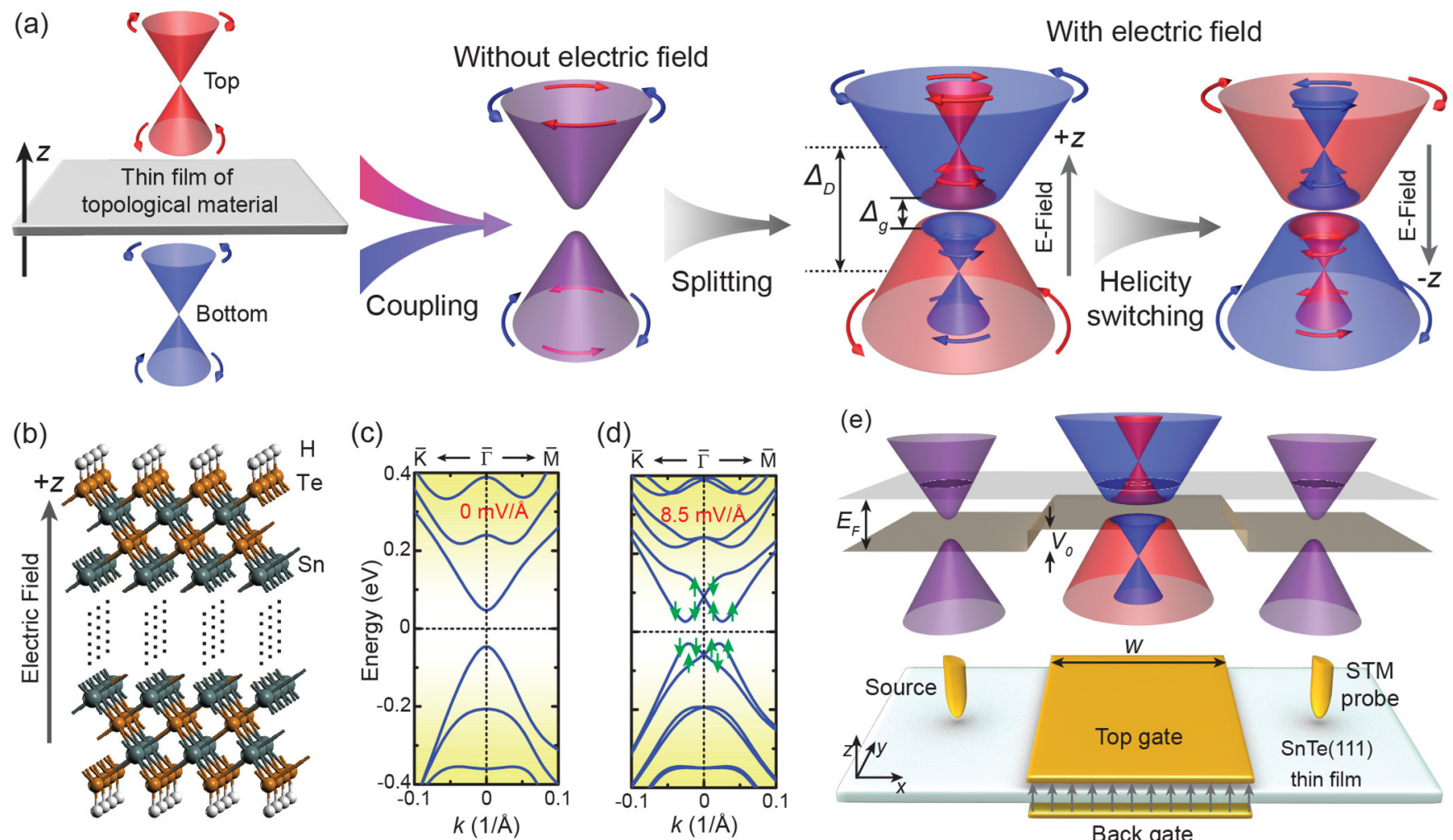

(d)

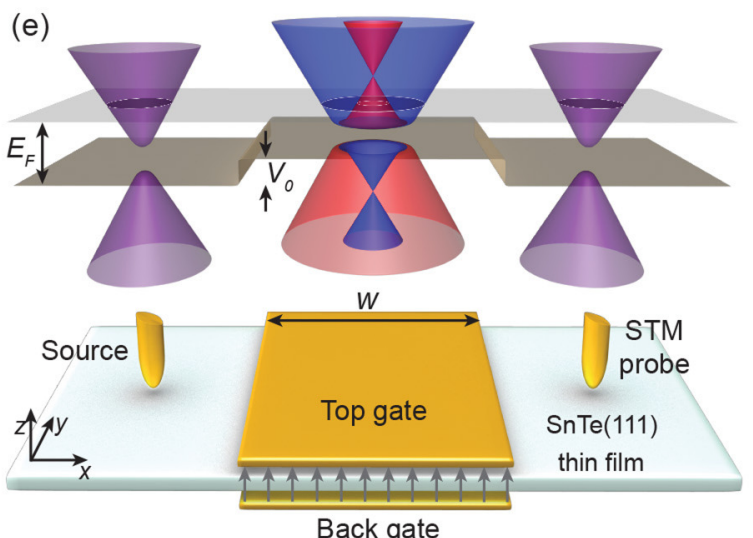

FIG. 2. (Color online) (a) A simplified model of electrically tuned band evolution in a thin film of topological material, where the helicity degree of freedom in the system can be controlled by a perpendicular electric field. The band gap is $\Delta_{g}$ and the energy splitting between Dirac points is $\Delta_{D}$. (b) Crystal structure of Te-terminated $\operatorname{SnTe}(111)$ thin film with hydrogen saturation. DFT-calculated band structure of a film with 25 atomic layers under (c) zero electric field and (d) a moderate electric field $E_{\text {ex }}=8.5 \mathrm{mV} / \AA$ which generates giant helicity splitting $\Delta_{D} \sim 0.15 \mathrm{eV}$. Also shown are the calculated in-plane helical spin textures (green arrows) for the electric field pointing in the $+z$ direction. (e) Schematic design of a dual-gated heterostructure tuned by the electric-field parameters $\delta$ and $V_{0}$. It may function as a helicity-based filter or flat lens for the incident helicity-degenerate Dirac electrons emitted from the source. The STM probe can be used to detect the transmitted helical electron waves. 
(a)

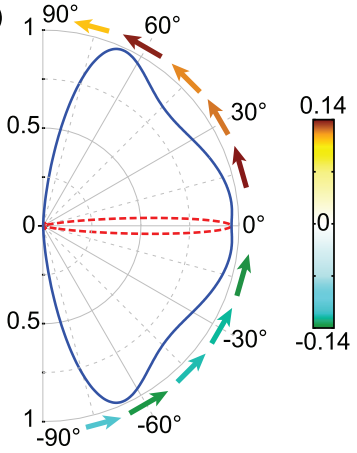

(b)

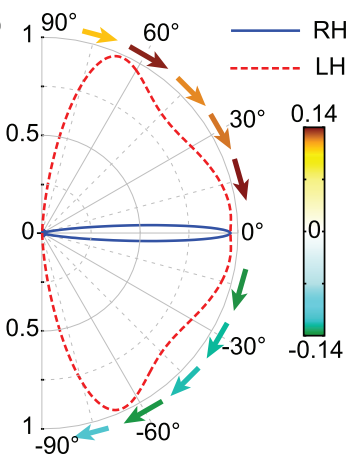

FIG. 3. (Color online) (a) Pronounced dichroism (i.e., helicity filtering) when the Fermi level crosses the upper Dirac point (i.e., $V_{0}=-23.9 \mathrm{meV}$ and $\delta=68 \mathrm{meV}$ ). The colorful arrows illustrate the spin orientations at different transmission angles, where the color scale gives the out-of-plane spin component. (b) Helicity switching induced by polarity inversion of the external electric field (i.e., $\delta=-68 \mathrm{meV}$ ), where both the transmission profiles and the in-plane spin orientations are switched. Note that the Fermi energy is $E_{F}=50 \mathrm{meV}$.

polarity of electric field is inverted [i.e., the sign of $\delta$ in Eq. (1) is changed], helicity configuration will reverse in both the conduction and valence bands, thus realizing helicity switching [Fig. 2(a)].

To explore helicity-dependent manipulation of Dirac fermions, a dual-gated heterostructure is exploited in the film [Fig. 2(e)]. We first investigate a Fabry-Pérot-type model containing a narrow dual-gated region with the width $w=$ $80 \mathrm{~nm}$, where the Fermi level in the dual-gated region is tuned to cross the upper Dirac point. For the helicitydegenerate incident electron wave, very strong directiondependent helicity-selective transmission (i.e., pronounced dichroism) is indicated in Fig. 3. In detail, for the potential height $V_{0}=E_{F}-\Delta_{D} / 2=-23.9 \mathrm{meV}$ with an upward electric field $(\delta=68 \mathrm{meV})$, the $\mathrm{RH}$ electron wave has very high transmission probability (up to $\sim 96 \%$ ) over a large angular range $\left(\sim \pm 70^{\circ}\right)$ with a Fabry-Pérot-like resonance profile, whereas a sharp transmission peak appears at normal incidence for the LH electron wave, leading to strong beam collimation [Fig. 3(a)]. Such pronounced helicity-selective transmission directly stems from the fact that the intrahelical scattering dominates over the interhelical one in the transmission events (see Fig. S4 in [39] for details), where the density of states for the LH electron state vanishes at the upper Dirac point. Moreover, helicity interference gives rise to the three-dimensional (3D) complex spin orientations along different transmission directions, illustrated by the colorful arrows in Fig. 3(a). Exactly at the normal incidence, the $\mathrm{RH}$ and $\mathrm{LH}$ electrons have the same transmission probability and thus the total transmitted spin polarization is zero. If the polarity of the electric field is inverted $(\delta=-68 \mathrm{meV}$ ), the transmission profiles for the $\mathrm{LH}$ and RH electrons will switch and the in-plane spin orientations of the transmitted wave will reverse [Fig. 3(b)].

Because the presence of a hole pocket in band structure could lead to negative refraction of electrons [41], we further consider a wide dual-gated region $(w=800 \mathrm{~nm})$ in the heterostructure to construct a helicity-dependent flat lens.

The Fermi level in the dual-gated region is tuned between the upper Dirac point and the conduction band minimum for the intraband tunneling (i.e., $E_{F}-\Delta_{g} / 2>V_{0}>E_{F}-\Delta_{D} / 2$ ). As such, RH hole and electron pockets with remarkably distinct Fermi wave vectors can coexist in the band structure due to giant helicity splitting. For simplicity, the inner RH hole pocket is set to have the same Fermi wave vector as the incident electron states (i.e., $V_{0}=10.2 \mathrm{meV}$ and $\delta=68 \mathrm{meV}$ ). An electron point source located at $(-a, 0)$ emits cylindrical helicity-degenerate electron wave shining on the interfaces. Based on the plane-wave expansion method [42], we calculate the spatial intensity distribution of transmitted electron waves for $a=100 \mathrm{~nm}$. Due to the prevailing intrahelical scattering (see Fig. S6 in [39]), only one perfect focal point at $x_{f}=2 w-a=1500 \mathrm{~nm}$ for the LH electron state is visible, whereas the $\mathrm{RH}$ electron state divergently passes through the flat lens [Fig. 4(a)], which exactly mimics the propagation of LH and RH photons through a slab of chiral metamaterials [2]. Because the RH hole (electron) state in the dual-gated region preferentially provides a scattering channel for the incident LH (RH) electron wave, the effective refractive indices can be given by $n_{\mathrm{LH}}=-1$ and $n_{\mathrm{RH}}=2.34$.

More interestingly, we also find the helicity-resolved negative birefraction effect, which is unique to Dirac fermions and has no direct counterpart in photonic chiral metamaterials. In this case, as the potential height increases to generate the interband tunneling (i.e., $V_{0}>E_{F}+\Delta_{D} / 2$ ), two hole pockets with opposite helicity simultaneously lie in the valence band. The inner LH hole pocket is set to have the same Fermi wave vector as the incident electron state (i.e., $V_{0}=162.5 \mathrm{meV}$ and $\delta=68 \mathrm{meV}$ ). Our results show that one more new focal region will appear due to helicity-dependent negative birefraction [Fig. 4(b)]. Because the intrahelical scattering is still dominant (see Fig. S8 in [39]), the effective refractive indices can be given by $n_{\mathrm{LH}}=-4.34$ and $n_{\mathrm{RH}}=-1$. The RH electrons can thus be perfectly focused at $x_{f}=1500 \mathrm{~nm}$, while the LH electrons rejoin near the classical cusp at $x_{c}=\left(1-1 / n_{\mathrm{LH}}\right) w-a=884.3 \mathrm{~nm}$, showing an intensity interference pattern in Fig. 4(b).

In Figs. 4(c)-4(e), we show the real-space projection of helicity interference of the focused LH or RH electron flows, characterized by the 3D complex spin distributions in the vicinity of the focal regions. Due to the interference of helical electron waves [i.e., Fig. 4(c) for the LH electrons and Fig. 4(e) for the RH electrons], the in-plane spin orientations will reverse near the focal point at $x_{f}=1500 \mathrm{~nm}$. Near the cusp at $x_{c}=884.3 \mathrm{~nm}$ [Fig. 4(d)], complex spin distribution for the LH electrons emerges in light of the negative birefraction. However, the antisymmetry of the out-of-plane spin patterns about the "optical axis" (i.e., the $x$ axis) remains unchanged in Figs. 4(c)-4(e), providing an additional signature of the helicity interference in real space. Upon the polarity inversion of the electric field $(\delta<0)$, all the in-plane spin orientations shown in Figs. 4(c)-4(e) will reverse for helicity switching operation.

Experimentally, the Fermi level can be tuned by adjusting $\mathrm{Pb}$ content in the $\mathrm{Pb}_{1-x} \mathrm{Sn}_{x} \mathrm{Te}(111)$ thin film grown by molecular beam epitaxy (MBE) [31]. The intensity and spin distributions can be measured by the scanning tunneling microscopy (STM) with nanoscale resolution [e.g., see Fig. 2(e)]. To suppress the wavelength deviation of Dirac fermions due 


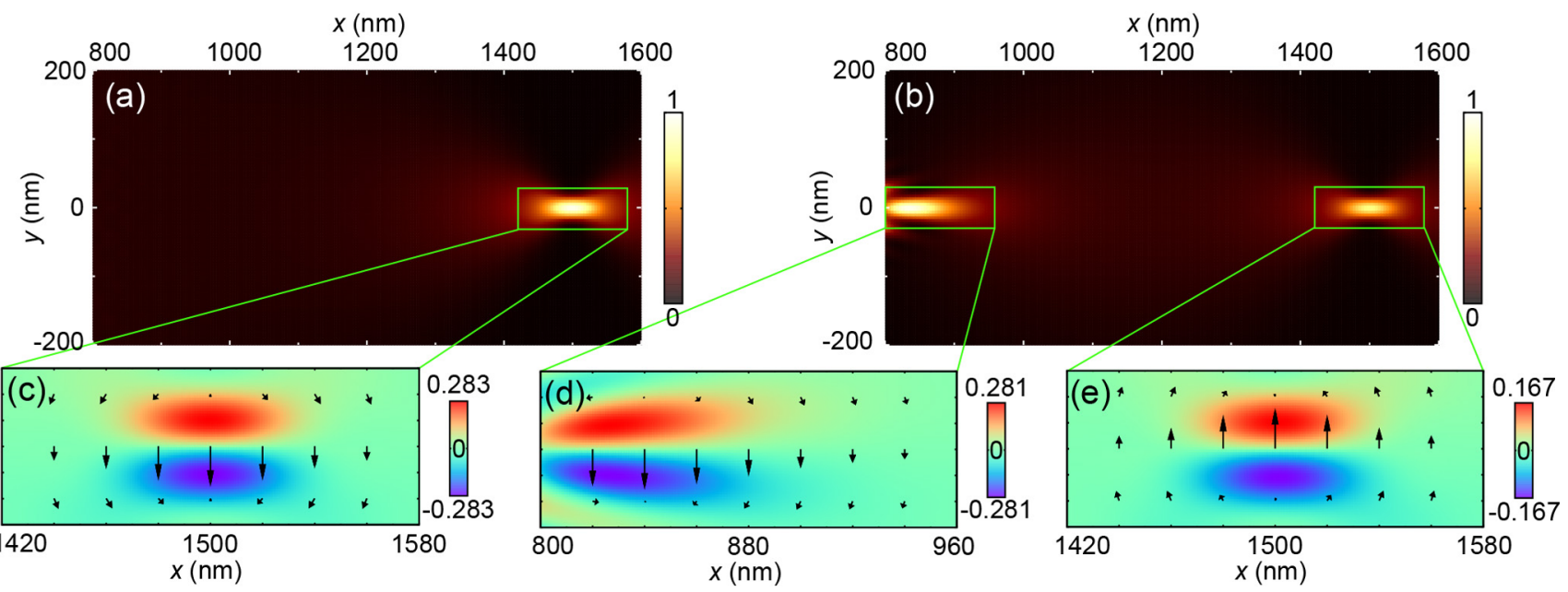

FIG. 4. (Color online) Helicity-dependent focusing through a flat lens $(w=800 \mathrm{~nm})$. An electron point source is placed at $(-100 \mathrm{~nm}, 0)$ and the Fermi energy is $E_{F}=50 \mathrm{meV}$. (a) and (b) Gate-tuned single and double focusing through the flat lens, depicted by the normalized maximum intensity of the transmitted waves. (c)-(e) Complex spin distributions near the focal regions due to helicity interference. The black arrows and the rainbow color scale indicate the in-plane and out-of-plane spin components, respectively.

to thermal excitation, low temperature is usually required. For $E_{F}=50 \mathrm{meV}$ used in our theoretical calculations, a proper temperature range can be estimated by $T \ll E_{F} / k_{B} \approx$ $580 \mathrm{~K}$, which can be easily achieved with present cryogenic techniques. Recently, a coherence length about $500 \mathrm{~nm}$ has been observed experimentally in SnTe thin films, which may be further enhanced by improving the MBE growth conditions [43]. Note that our system is highly tunable. Thus, by rescaling the parameters, the focusing effect could be realized in the range of hundreds of nanometers [44], where the decoherence effect can be negligible. In addition, our DFT calculations also show that the atomic step edges on the SnTe(111) thin films with surface functionalization may provide another viable avenue to observe the helicity-dependent transport behaviors of Dirac fermions [31,39,45].

In summary, like the spin and valley degrees of freedom forming the basis for spintronics and valleytronics, the idea of exploiting the helicity degree of freedom of Dirac fermions as information carriers could open up a promising field for helicity-based electronics (or, in brief, helictronics). By analogy with LH and RH photons in chiral metamaterials, we have verified such idea in thin films of topological materials for helicity-resolved manipulation of Dirac fermions. Our findings may shed new light on the control strategies for Dirac fermions with high tunability and scalability.

We thank Liang Fu, Zuxin Jin, and Xiaohang Sun for helpful discussions. We acknowledge support from the National Natural Science Foundation of China (Grants No. 11204154 and No. 11334006) and the Ministry of Science and Technology of China (Grants No. 2011CB921901 and No. 2011CB606405). L.Z. would also like to thank financial support by the Open Research Fund Program of the State Key Laboratory of Low-Dimensional Quantum Physics (No. KF201408).

L.Z., J.W., and J.L. contributed equally to this work.
[1] S. Tretyakov, I. Nefedov, A. Sihvola, S. Maslovski, and C. Simovski, J. Electromagn. Waves Appl. 17, 695 (2003).

[2] J. B. Pendry, Science 306, 1353 (2004).

[3] J. K. Gansel, M. Thiel, M. S. Rill, M. Decker, K. Bade, V. Saile, G. von Freymann, S. Linden, and M. Wegener, Science 325, 1513 (2009).

[4] S. Zhang, Y.-S. Park, J. Li, X. Lu, W. Zhang, and X. Zhang, Phys. Rev. Lett. 102, 023901 (2009).

[5] M. E. Peskin and D. V. Schroeder, An Introduction to Quantum Field Theory (Westview, Boulder, CO, 1995).

[6] X.-L. Qi and S.-C. Zhang, Rev. Mod. Phys. 83, 1057 (2011).

[7] M. Z. Hasan and C. L. Kane, Rev. Mod. Phys. 82, 3045 (2010).

[8] J. E. Moore, Nature (London) 464, 194 (2010).

[9] L. Fu, Phys. Rev. Lett. 106, 106802 (2011).

[10] T. H. Hsieh, H. Lin, J. Liu, W. Duan, A. Bansil, and L. Fu, Nat. Commun. 3, 982 (2012).
[11] J. Linder, T. Yokoyama, and A. Sudbø, Phys. Rev. B 80, 205401 (2009).

[12] H.-Z. Lu, W.-Y. Shan, W. Yao, Q. Niu, and S.-Q. Shen, Phys. Rev. B 81, 115407 (2010).

[13] C.-X. Liu, H. J. Zhang, B. Yan, X.-L. Qi, T. Frauenheim, X. Dai, Z. Fang, and S.-C. Zhang, Phys. Rev. B 81, 041307(R) (2010).

[14] O. V. Yazyev, J. E. Moore, and S. G. Louie, Phys. Rev. Lett. 105, 266806 (2010).

[15] M. Kim, C. H. Kim, H.-S. Kim, and J. Ihm, Proc. Natl. Acad. Sci. USA 109, 671 (2012).

[16] T. Zhang, J. Ha, N. Levy, Y. Kuk, and J. Stroscio, Phys. Rev. Lett. 111, 056803 (2013).

[17] Y. Zhang et al., Nat. Phys. 6, 584 (2010).

[18] H. Jin, J.-H. Song, and A. J. Freeman, Phys. Rev. B 83, 125319 (2011).

[19] Y. Tanaka, Z. Ren, T. Sato, K. Nakayama, S. Souma, T. Takahashi, K. Segawa, and Y. Ando, Nat. Phys. 8, 800 (2012). 
[20] P. Dziawa et al., Nat. Mater. 11, 1023 (2012).

[21] S. Y. Xu et al., Nat. Commun. 3, 1192 (2012).

[22] Y. Okada et al., Science 341, 1496 (2013).

[23] I. Zeljkovic et al., Nat. Phys. 10, 572 (2014).

[24] J. Liu, T. H. Hsieh, P. Wei, W. Duan, J. Moodera, and L. Fu, Nat. Mater. 13, 178 (2014).

[25] S. Safaei, P. Kacman, and R. Buczko, Phys. Rev. B 88, 045305 (2013).

[26] J. Liu, W. Duan, and L. Fu, Phys. Rev. B 88, 241303(R) (2013).

[27] J. Wang, J. Liu, Y. Xu, J. Wu, B.-L. Gu, and W. Duan, Phys. Rev. B 89, 125308 (2014).

[28] Y. Tanaka, T. Shoman, K. Nakayama, S. Souma, T. Sato, T. Takahashi, M. Novak, K. Segawa, and Y. Ando, Phys. Rev. B 88, 235126 (2013).

[29] C. M. Polley et al., Phys. Rev. B 89, 075317 (2014).

[30] A. A. Taskin, F. Yang, S. Sasaki, K. Segawa, and Y. Ando, Phys. Rev. B 89, 121302(R) (2014).

[31] C. Yan et al., Phys. Rev. Lett. 112, 186801 (2014).

[32] G. Kresse and J. Furthmüller, Comput. Mater. Sci. 6, 15 (1996).

[33] G. Kresse and J. Furthmüller, Phys. Rev. B 54, 11169 (1996).

[34] P. E. Blöchl, Phys. Rev. B 50, 17953 (1994).

[35] J. P. Perdew and Y. Wang, Phys. Rev. B 45, 13244 (1992).

[36] L. Bengtsson, Phys. Rev. B 59, 12301 (1999).

[37] B. Meyer and D. Vanderbilt, Phys. Rev. B 63, 205426 (2001).

[38] To study $\mathrm{SnTe}(111)$ thin films, first-principles DFT calculations are performed by the Vienna $a b$ initio simulation package $[32,33]$ using the projector augmented wave method [34] and the Perdew-Wang-91 [35] generalized gradient approximation exchange-correlation functional. The plane-wave basis with an energy cutoff of $250 \mathrm{eV}$ and the $\Gamma$-centered $k$-point meshes are adopted. SnTe(111) thin films of different thicknesses are calculated by using slab models with dangling bonds saturated by hydrogen [27]. The slab configurations are fully relaxed with remaining forces less than $5 \mathrm{meV} / \AA$. A dipole correction is applied in the slab calculations to eliminate the artificial interactions between periodic images [36,37]. The spin-orbit coupling is included when we calculate the band structures.

[39] See Supplemental Material at http://link.aps.org/supplemental/ 10.1103/PhysRevB.92.041408 for details of the band structures and the transport simulations.

[40] R. Winkler, Spin-Orbit Coupling Effects in Two-Dimensional Electron and Hole Systems (Springer, Berlin, 2003).

[41] V. V. Cheianov, V. Fal'ko, and B. L. Altshuler, Science 315, 1252 (2007).

[42] G. Cincotti, F. Gori, M. Santarsiero, F. Frezza, F. Furno, and G. Schettini, Opt. Commun. 95, 192 (1993).

[43] B. A. Assaf, F. Katmis, P. Wei, B. Satpati, Z. Zhang, S. P. Bennett, V. G. Harris, J. S. Moodera, and D. Heiman, Appl. Phys. Lett. 105, 102108 (2014).

[44] Based on the classical trajectory, we can estimate the positions of focal regions. For example, the width of the flat lens can be reduced to $w=300 \mathrm{~nm}$, the point source is moved to the location at $(-50 \mathrm{~nm}, 0)$ (i.e., $a=50 \mathrm{~nm}$ ), and the Fermi level is increased to $E_{F}=70 \mathrm{meV}$. For $V_{0}=204.9 \mathrm{meV}$, the RH electrons can be perfectly focused at $x_{f}=2 w-a=550 \mathrm{~nm}$ where $n_{\mathrm{RH}}=-1$, while the LH electrons converge near the classical cusp at $x_{c}=$ $\left(1-1 / n_{\mathrm{LH}}\right) w-a=345.7 \mathrm{~nm}$ where $n_{\mathrm{LH}}=-3.13$.

[45] J. Seo, P. Roushan, H. Beidenkopf, Y. S. Hor, R. J. Cava, and A. Yazdani, Nature (London) 466, 343 (2010). 